\title{
Characterization of the Initial Enzymatic Steps of
}

\section{Barbamide Biosynthesis}

Patricia M. Flatt ${ }^{\dagger}$, Susan J. O'Connell ${ }^{\ddagger}$,Kerry L. McPhail ${ }^{\dagger}$, Gloria Zeller ${ }^{\dagger}$, Christine L. Willis ${ }^{\ddagger}$, David H. Sherman ${ }^{\S}$, and William H. Gerwick $*^{\perp^{\perp}}$

College of Pharmacy, Oregon State University, Corvallis, OR 97331

School of Chemistry, University of Bristol, Bristol, BS8 ITS UK

Life Sciences Institute, Department of Medicinal Chemistry, Department of Chemistry, and Department of Microbiology and Immunology, University of Michigan, Ann Arbor, MI 48109

Scripps Institution of Oceanography and Skaggs School of Pharmacy and Pharmaceutical Sciences, University of California San Diego, La Jolla, CA 92093

S1. Panels of selected carbon atom intensities in barbamide produced during supplementation with $\left[6-{ }^{13} \mathrm{C}\right](2 \mathrm{~S}, 4 \mathrm{~S})-5,5-$ dichloroleucine, normalized to various carbon signals in the natural abundance spectrum. The average normalized percent enrichment at C-1 (e.g average of comparisons with C-2, C-3, C-4, C-7, C-8 and $\mathrm{OCH}_{3}$ ) is $604 \%$.

S2. LC-MS data for ${ }^{13} \mathrm{C}$ labeled barbamide (1): a) Total Ion Count, b) UV profile at $254 \mathrm{~nm}, \mathrm{c}$ ) UV profile at $230 \mathrm{~nm}, \mathrm{~d}$ ) Positive mode ESI mass spectrum.

S3. $\quad$ LC-MS data for dechlorobarbamide (2): a) Total Ion Count, b) Positive mode ESI mass spectrum.

S4. LC-MS data for didechlorobarbaleucamide B (7): a) Total Ion Count, b) Positive mode ESI mass spectrum, c) HR-ESIMS data.

S5. $\quad$ NMR data (DMSO-d6; $300 \mathrm{MHz}$ ) for ${ }^{13} \mathrm{C}$ labeled didechlorobarbaleucamide B (7): a) ${ }^{13} \mathrm{C}$ NMR spectrum with inset of upfield region showing two enriched ${ }^{13} \mathrm{C}$ methyl signals (C-10 and C-12); b) ${ }^{1} \mathrm{H}$ NMR spectrum. 
<smiles>CCC(C)(Cl)CC(=CC(=O)N(C)[C@H](Cc1ccccc1)c1nccs1)OC</smiles>

Barbamide $\left(\mathrm{C}_{20} \mathrm{H}_{23}{ }^{35} \mathrm{Cl}_{3} \mathrm{~N}_{2} \mathrm{O}_{2} \mathrm{~S}\right)$

Exact Mass: 460.05

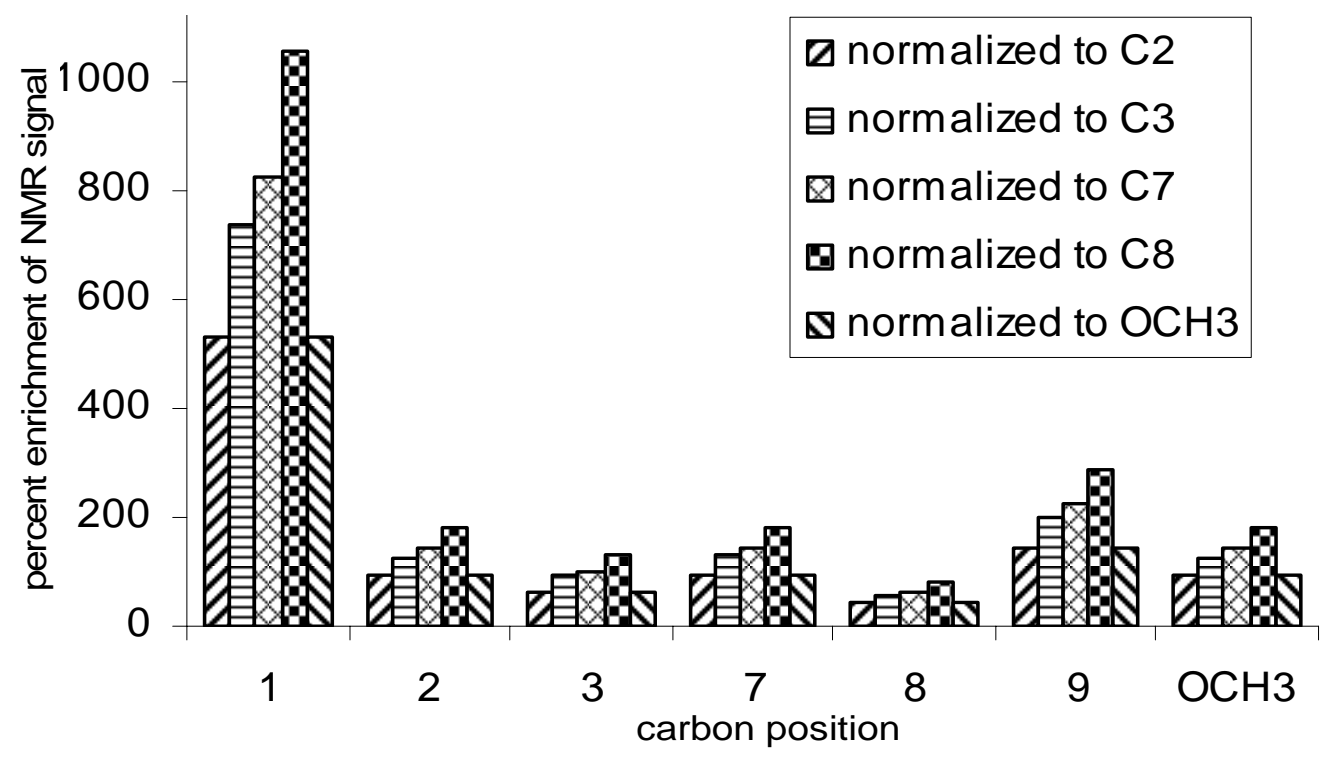

S1. Panels of selected carbon atom intensities for barbamide produced during supplementation with [6$\left.{ }^{13} \mathrm{C}\right](2 S, 4 S)$-5,5-dichloroleucine, normalized to various carbon signals in the natural abundance spectrum. The average normalized percent enrichment at C-1 (e.g average of comparisons with C-2, C-3, C-4, C-7, C-8 and $\mathrm{OCH}_{3}$ ) is $604 \%$. 

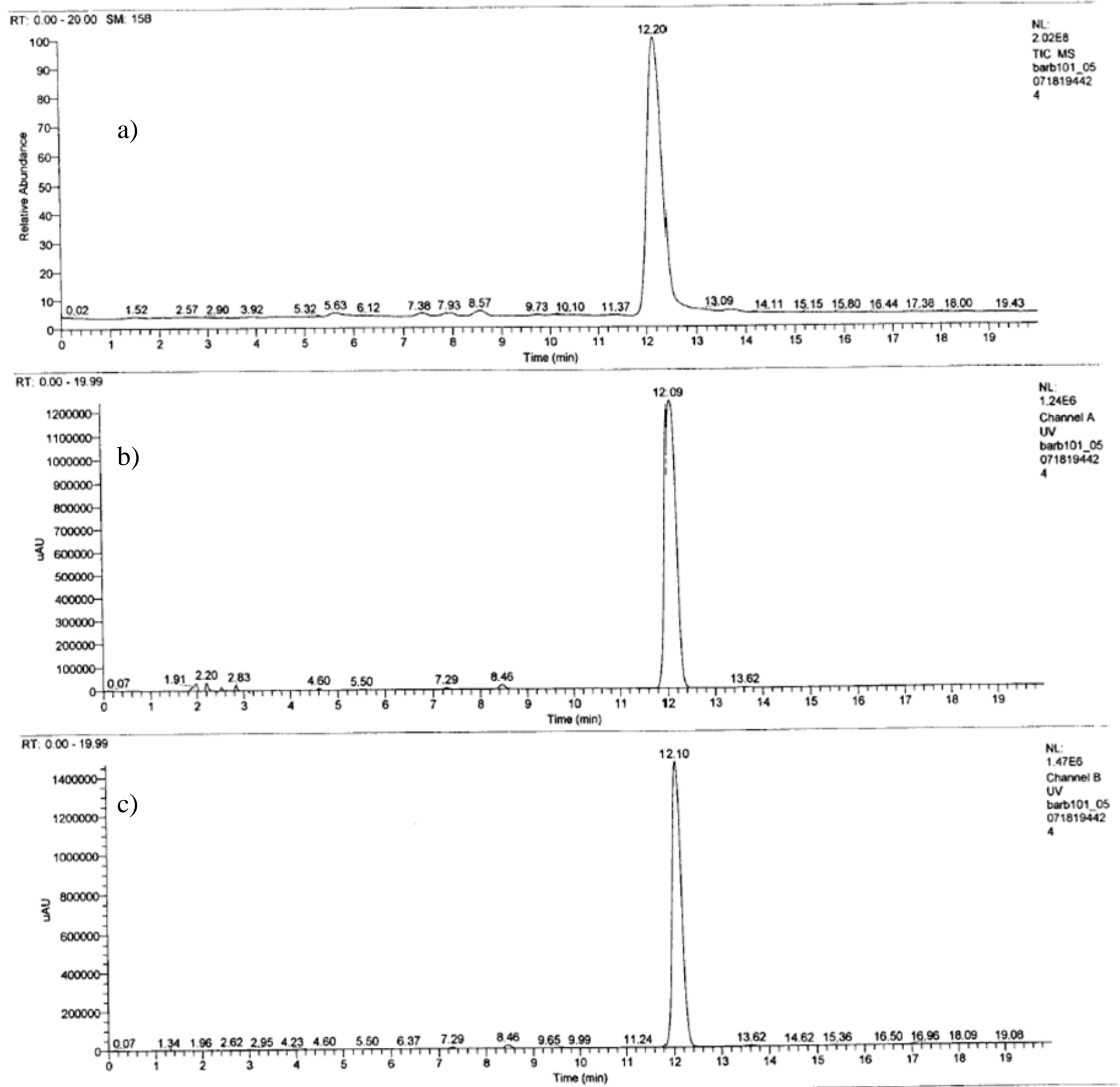

barb101 050718194424 \#745-766 RT: 12.04-12.32 AV: 22 NL: 5.47E7

$\mathrm{T}$ : + c ESI Full ms [ 150.00-1000.00]

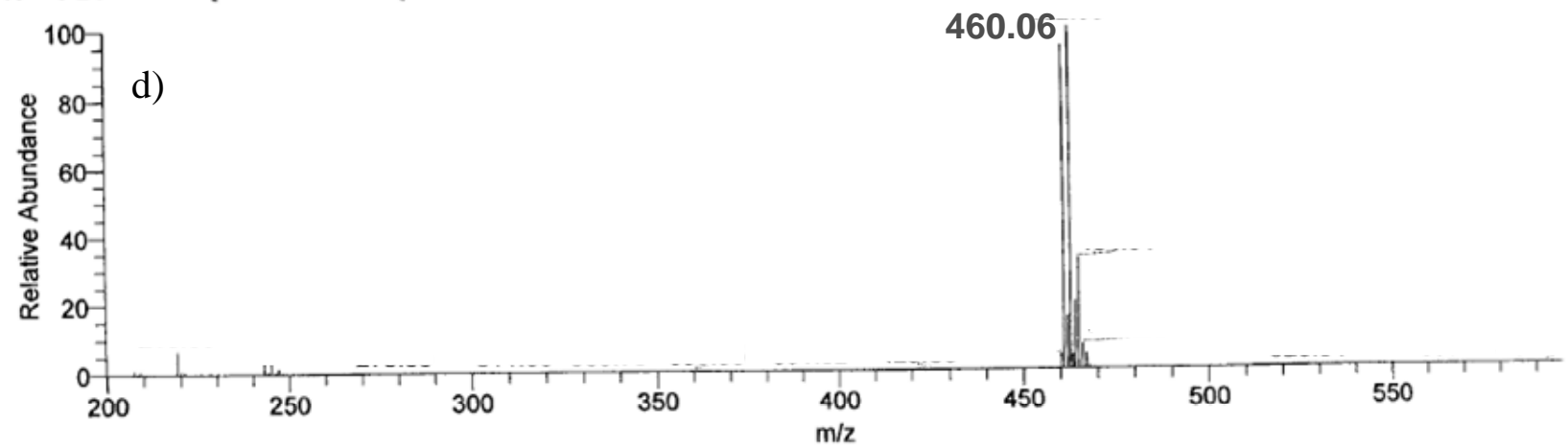

S2. LC-MS data for ${ }^{13} \mathrm{C}$ labeled barbamide: a) Total Ion Count, b) UV profile at $254 \mathrm{~nm}$, c) UV profile at $230 \mathrm{~nm}$, d) Positive mode ESI mass spectrum. 

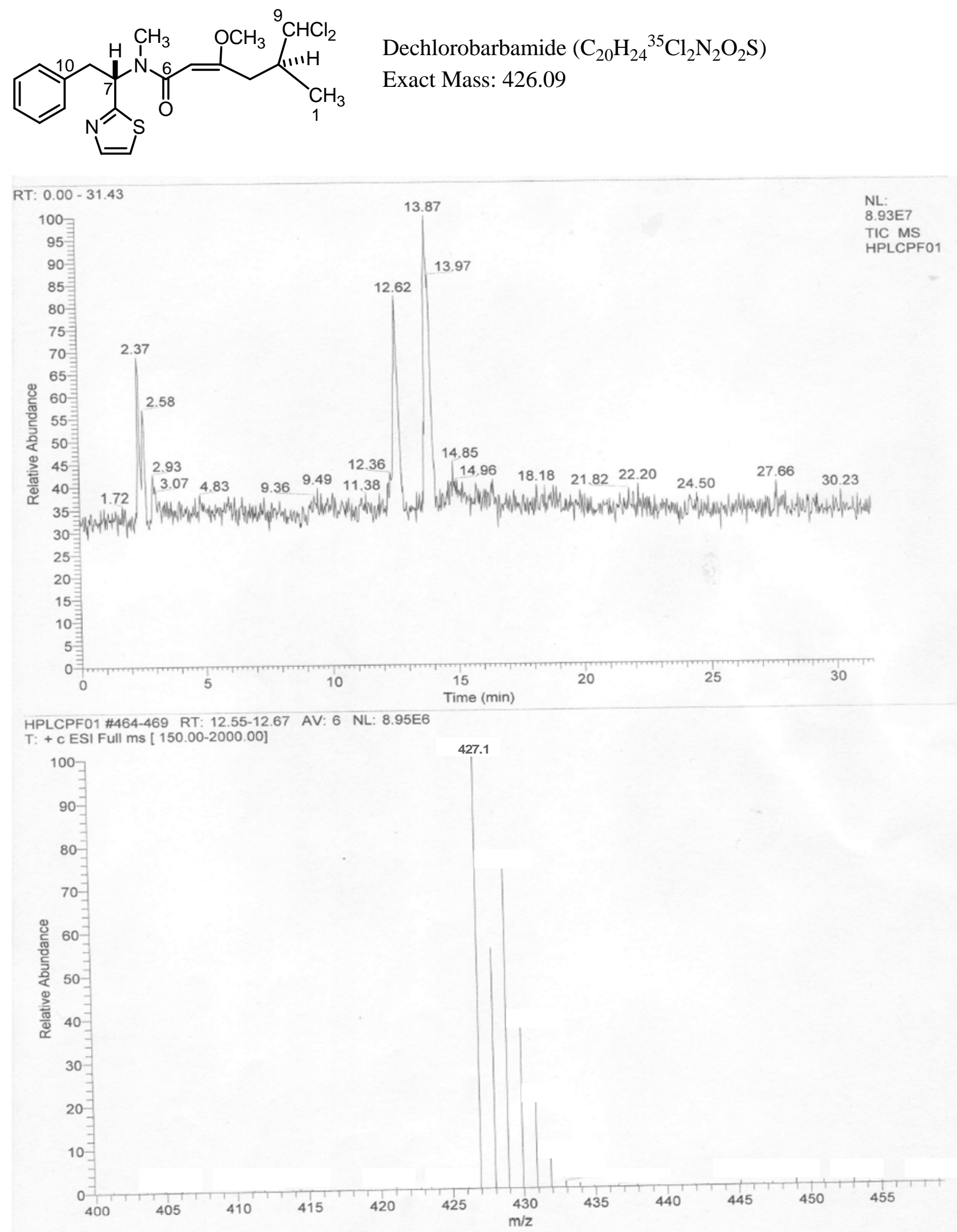

S3. LC-MS data for dechlorobarbamide (2): a) Total Ion Count, b) Positive mode ESI mass spectrum. 


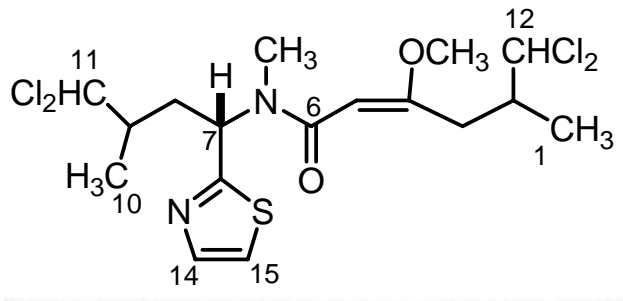

Didechlorobarbaleucamide B $\left(\mathrm{C}_{17} \mathrm{H}_{24}{ }^{35} \mathrm{Cl}_{4} \mathrm{~N}_{2} \mathrm{O}_{2} \mathrm{~S}\right)$

Exact Mass: 460.03

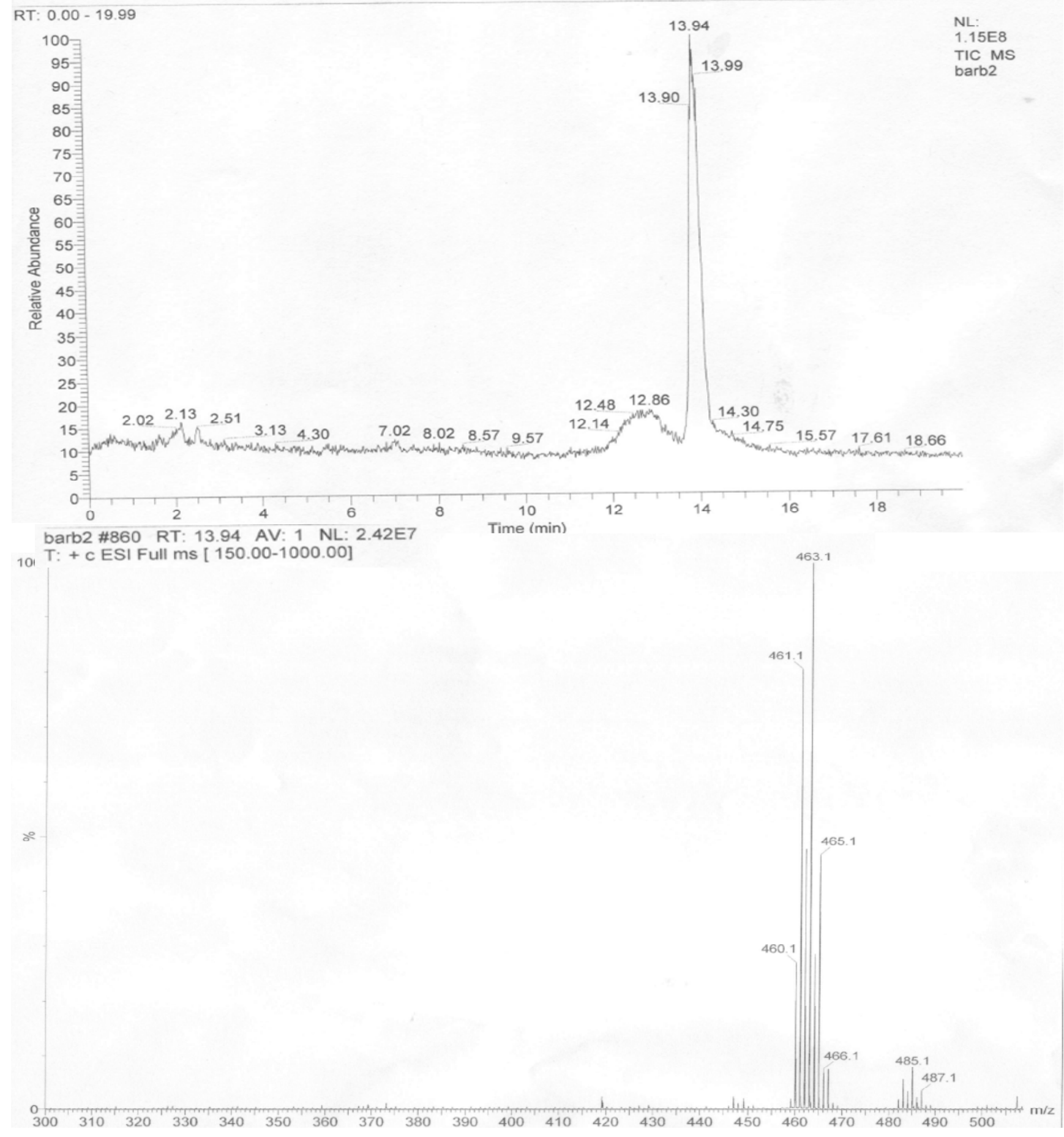

c) HR-ESIMS obsd $\mathrm{M}^{+} \mathrm{m} / \mathrm{z} 460.0300$ (calcd for $\mathrm{C}_{17} \mathrm{H}_{24}{ }^{35} \mathrm{Cl}_{4} \mathrm{~N}_{2} \mathrm{O}_{2} \mathrm{~S}, 460.0313$ )

S4. LC-MS data for didechlorobarbaleucamide B (7): a) Total Ion Count, b) Positive mode ESI mass spectrum, c) HR-ESIMS data. 

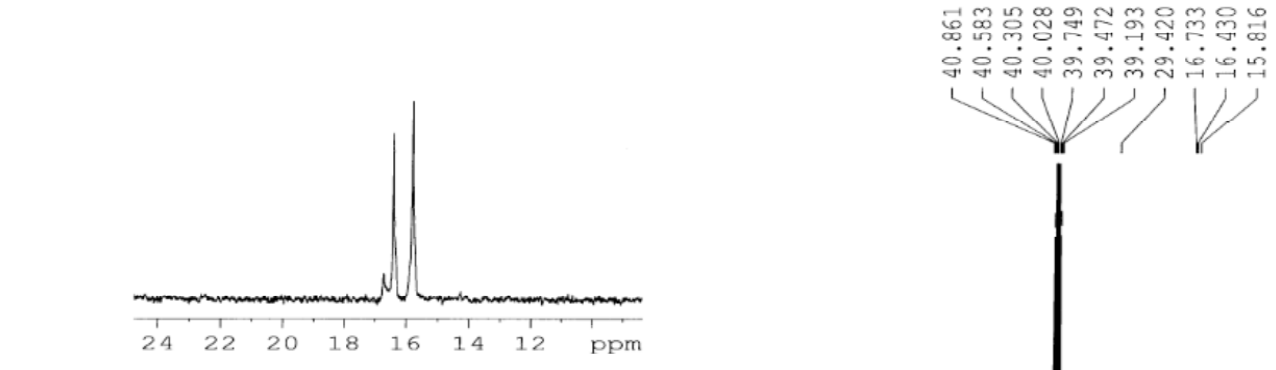

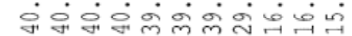
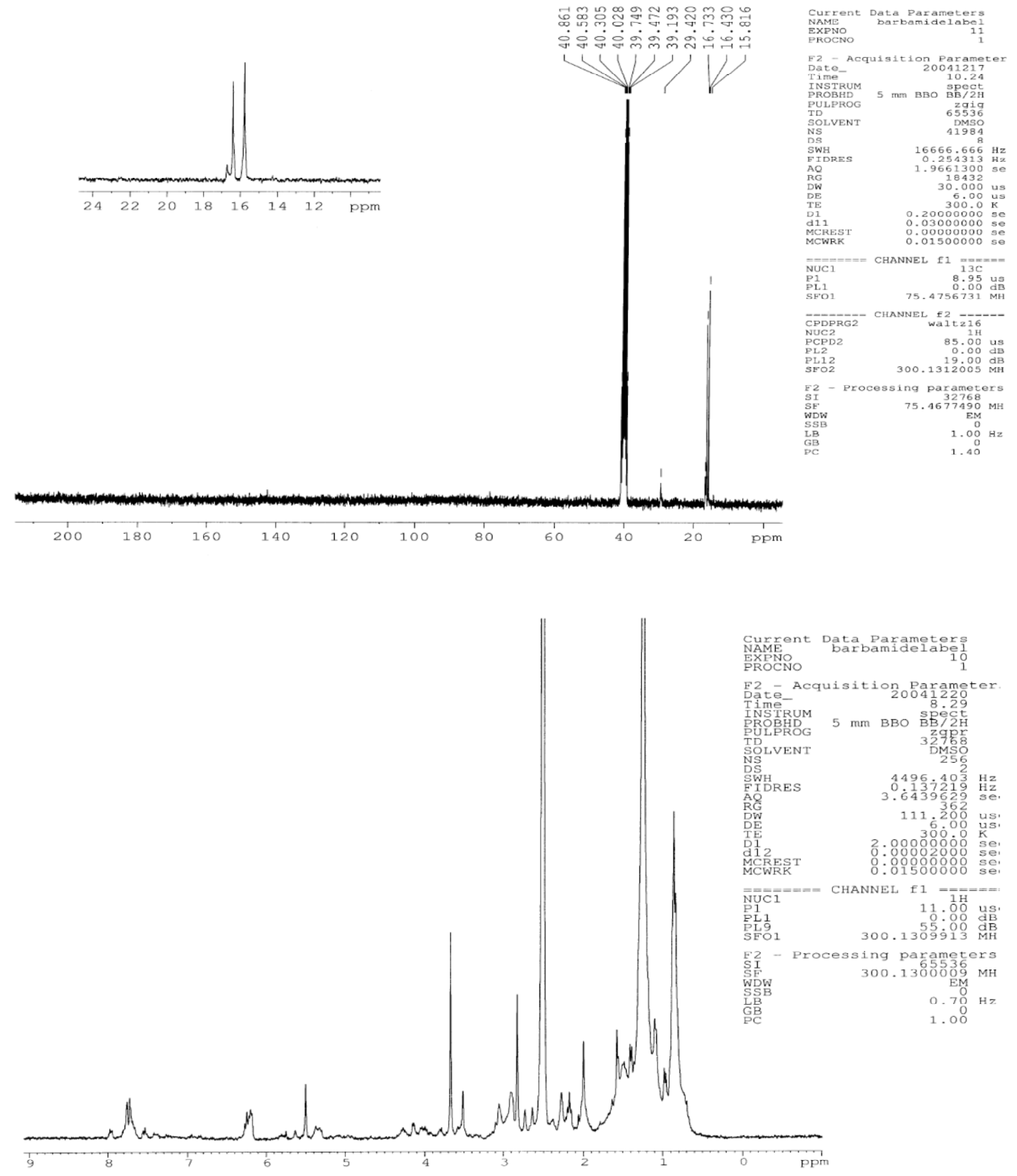

S5. NMR data (DMSO- $d_{6} ; 300 \mathrm{MHz}$ ) for ${ }^{13} \mathrm{C}$ labeled didechlorobarbaleucamide B (7): a) ${ }^{13} \mathrm{C}$ NMR spectrum with inset of upfield region showing two enriched ${ }^{13} \mathrm{C}$ methyl signals (C-1 and C-12); b) ${ }^{1} \mathrm{H}$ NMR spectrum. 